\title{
Nonlinear frequency shifts in quasispherical-cap shells: Pitch glide in Chinese gongs
}

\author{
N. H. Fletcher \\ Institute of Physical Sciences, CSIRO, Limestone Avenue, Canberra ACT 2602, Australia
}

(Received 6 June 1985; accepted for publication 3 September 1985)

\begin{abstract}
The large-amplitude vibrational behavior of a shallow spherical-cap shell is investigated theoretically using simple arguments. The results of this approximate analysis are expressed in the form of appealingly simple nondimensional quantities. It is shown that the frequency of the fundamental mode of such a shell falls by as much as $50 \%$ as the vibration amplitude is increased to approximately the shell depth. For even larger amplitudes the frequency rises, and it exceeds the small-amplitude frequency when the amplitude is more than about twice the shell depth. The influence of shell thickness is considered and increasing thickness is shown to decrease the frequency shift. This analysis is shown to account for the pitch glide behavior of certain Chinese opera gongs.
\end{abstract}

PACS numbers: 43.40.Ey, 43.40.Ga, 43.75.Kk

\section{INTRODUCTION}

In a recent paper in this Journal, Rossing and Fletcher ${ }^{1}$ reported an experimental study of the small-amplitude and large-amplitude behavior of the vibration of Chinese opera gongs. These gongs, which are circular and have the sort of cross section shown in Fig. 1, are acoustically interesting because they exhibit contrary pitch glides. The larger gongs, the central striking surface of which is flat, glide downward in pitch by as much as three semitones after being struck. The smaller gongs, which have a very slightly domed striking surface, glide upward in pitch by as much as two semitones.

The experimental study investigated several possibilities and concluded that the glide direction was probably determined by a combination of the effects of curvature and of internal stress. Only a qualitative discussion was given.

In retrospect, it turns out to be quite simple to give a quantitative analysis of the large-amplitude behavior of gongs such as these and, since the phenomena are aurally striking, easily demonstrated, and applicable to a variety of related structures, it seems worthwhile to present the discussion briefly here. While the analysis glosses over many of the details in the interests of simplicity, this is in many ways an advantage, and the treatment does represent a significant extension of more rigorous developments published in the literature. ${ }^{2.3}$

\section{ANALYSIS}

Even for quite large vibration amplitudes the motion of the gong is confined largely to the central circular section.' This is not unexpected since, for axisymmetric vibrations such as are excited by a central blow from a soft hammer, the conical shell surrounding the striking surface is rather stiff. ${ }^{4}$ The function of the conical flange and the outer cylindrical rim is thus largely to support the central vibrating section with its edge nearly rigidly clamped. For a freely suspended gong, the outer flange section moves nearly like a rigid body, ${ }^{4}$ at least for the lower vibrational modes, and contributes significantly to the sound radiation, rather as does the soundboard of a piano. The mass of this outer section is typically five to ten times that of the central section, and consideration of its motion has a small effect on the calculated mode frequencies. Referred to axes fixed in the flange section, however, the mode shapes on the central part of the gong are very nearly the same as if the flange were of infinite mass.

We, therefore, simplify our discussion by considering the active part of the gong to consist of a quasispherical domed shell with its edge rigidly clamped. The nonzero mechanical admittance of the conical flange need not concern us since we will not be concerned with calculating precise resonance frequencies. The case of a gong with a flat central section is clearly included as a limiting case in the derivation.

In the simplest treatment, we can neglect the flexural stiffness of the shell and consider its elastic rigidity to be provided by the membrane forces arising from the geometri-
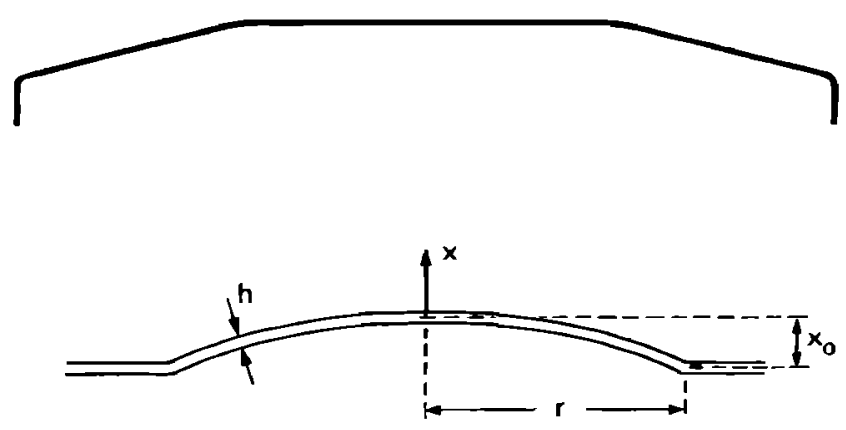

FIG. 1. The profile of a Chinese opera gong in cross section. Gongs range from 20 to $30 \mathrm{~cm}$ in diameter. Some of the gongs, typically the larger ones, have a fiat central section and glide downwards in pitch after being struck. The smaller gongs have a domed central section and glide upwards in pitch. Below is shown the coordinate system used to analyze the behavior of a domed gong. 
cal constraint of its curvature. If we consider just the fundamental vibration mode, with no nodal lines or circles, then we can associate with this a generalized coordinate $x$ which measures the normal displacement away from equilibrium of the central point of the shell. In this coordinate system, shown in Fig. 1, the plane through the rim of the shell lies at $x=-x_{0}$, and if the displacement is such that $x=-x_{0}$, then the motion of the shell has brought it to a plane configuration.

If $F_{0}(x)$ is a generalized force upon the shell, distributed so as to distort it in the form of the fundamental vibration mode (this force is provided by acceleration during the vibrational motion), then we must be able to write, correct to order $x^{4}$,

$$
F_{0}(x)=a\left(x+x_{0}\right)-b\left(x+x_{0}\right)^{3},
$$

the form of this function following from the existence of a configuration of unstable equilibrium $F_{0}\left(-x_{0}\right)=0$ for the flat configuration of the shell, and the requirement that $F_{0}(x)$ be antisymmetric about $x=-x_{0}$. Since $x=0$ is a configuration of stable equilibrium, $F_{0}(0)=0$ and, hence,

$$
a=b x_{0}^{2} .
$$

The general form of the force/displacement curve is thus as shown in Fig. 2.

We should allow, however, for the fact that the shell has appreciable rigidity because of its thickness, and this contributes a further term to the restoring force so that

$$
F(x)=F_{0}(x)-c x,
$$

the curve for which is also shown in Fig. 2. The nonlinearity associated with the stiffness term is very much less than that arising from the membrane forces and it can reasonably be neglected.

Using (1) $(3)$ then and assuming an effective mass $m$ to be associated with this mode, the equation of motion is

$$
m \ddot{x}=-b x\left(x+x_{0}\right)\left(x+2 x_{0}\right)-c x .
$$

If we consider just the linear terms on the right side, we can define the small-amplitude frequency limit $\omega_{0}$ to be given by

$$
\omega_{0}^{2}=\left(2 b x_{0}^{2}+c\right) / m \text {, }
$$

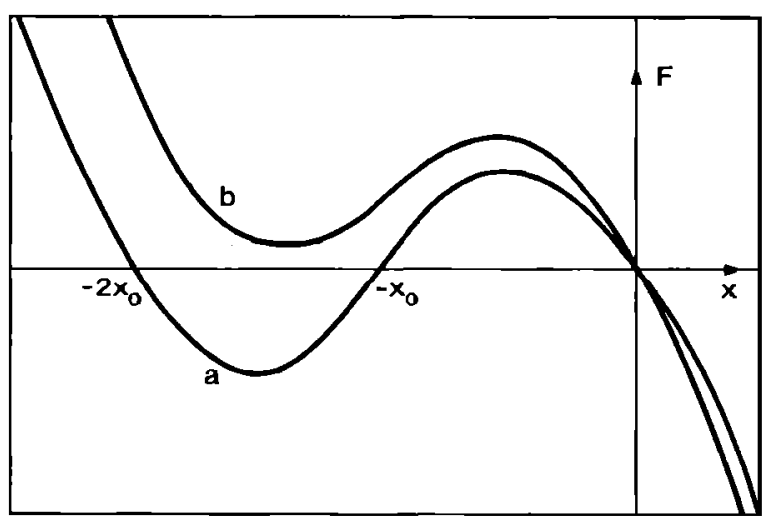

F1G. 2. The relationship between force $F$ and displacement $x$ for a sphericalcap shell. Curve a describes the behavior of an extremely thin shell whichcan be everted, while curve $b$ describes a shell of finite thickness which has only one equilibrium configuration. and Eq. (4) can then be written

$$
\ddot{x}+\omega_{0}^{2} x=-\left[\left(\omega_{0}^{2}-c / m\right) / 2 x_{0}^{2}\right]\left(x^{3}+3 x_{0} x^{2}\right)-2 k \dot{x},
$$

where we have included also a viscous damping term.

We can easily develop an approximate solution to this nonlinear differential equation by a simple extension of the method of slowly varying parameters. ${ }^{5}$ If we assume a solution of the form

$$
x=A \cos \psi+B,
$$

where

$$
\psi=\omega_{0} t+\phi
$$

and the displacement $B$, the amplitude $A$, and the phase $\phi$ are all regarded as slowly varying quantities constrained by the relation

$$
\dot{x}=-A \omega_{0} \sin \psi \text {, }
$$

then it is readily shown that, to a first approximation and correcting an error of sign in the reference,

$$
\begin{aligned}
& B \approx\left\langle\frac{1}{2 \pi \omega_{0}^{2}} \int_{0}^{2 \pi} g(A, B, \psi) d \psi\right\rangle, \\
& \dot{A} \approx-\left\langle\frac{1}{2 \pi \omega_{0}} \int_{0}^{2 \pi} g(A, B, \psi) \sin \psi d \psi\right\rangle, \\
& \dot{\phi} \approx-\left\langle\frac{1}{2 \pi A \omega_{0}} \int_{0}^{2 \pi} g(A, B, \psi) \cos \psi d \psi\right\rangle,
\end{aligned}
$$

where $g(A, B, \psi)$ is the right-hand side of (6) written in the form

$$
\begin{aligned}
g(A, B, \psi)= & -\left[\left(\omega_{0}^{2}-c / m\right) / 2 x_{0}^{2}\right]\left[(A \cos \psi+B)^{3}\right. \\
& \left.+3 x_{0}(A \cos \psi+B)^{2}\right]+2 k A \omega_{0} \sin \psi
\end{aligned}
$$

and the angle brackets $\langle>$ in (10)-(12) signify that only terms varying slowly in comparison with the frequency $\omega_{0}$ are to be retained.

\section{A. Thin shell approximation}

These equations can be solved quite generally, but we focus attention initially on the case of a very thin shell for which $c<m \omega_{0}^{2}$ so that the rigidity is provided essentially by the membrane forces. For this case, we find that, for $A \leqslant(2)$ $3)^{1 / 2} x_{0} \approx 0.8 x_{0}$,

$$
\begin{aligned}
& B \approx-x_{0} \pm\left[x_{0}^{2}-(3 / 2) A^{2}\right]^{1 / 2}, \\
& \dot{A} \approx-k A, \\
& \dot{\phi} \approx-(15 / 16)\left(A / x_{0}\right)^{2} \omega_{0},
\end{aligned}
$$

while for $A>(2 / 3)^{1 / 2} x_{0}, \dot{A}$ is still given by (15) but

$$
\begin{aligned}
& B \approx-x_{0}, \\
& \dot{\phi} \approx\left[(3 / 16)\left(A / x_{0}\right)^{2}-3 / 4\right] \omega_{0} .
\end{aligned}
$$

In either case, the value of $B$ gives the location of the effective centroid of the oscillation. Equation (15) shows that the motion is exponentially damped, while Eq. (16) or (18) gives the effective frequency $\omega$ through the relation

$$
\omega=\omega_{0}+\dot{\phi} \text {. }
$$

The behavior of $B$ and $\omega$ as functions of the amplitude $A$ is shown in Fig. 3. The frequency $\omega$ decreases quite sharply 

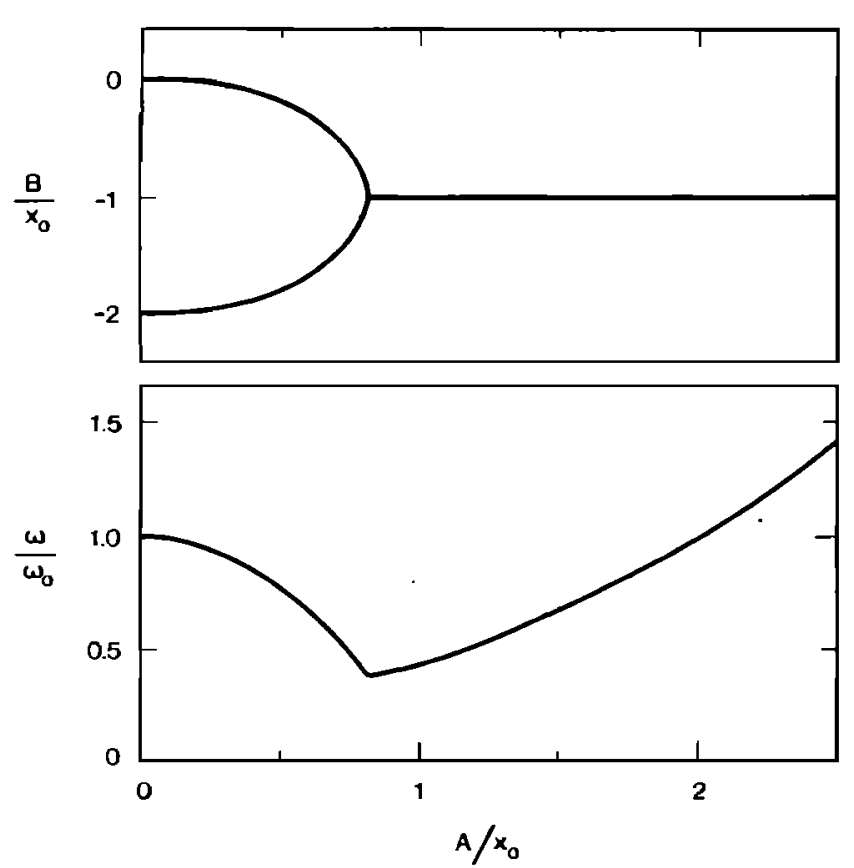

FIG. 3. Calculated motion center $B$ and frequency $\omega$ for vibrations of amplitude $A$ on a thin spherical-cap shell of dome height $x_{0}$. The normal mode frequency for small-amplitude vibrations is $\omega_{0}$.

with increasing amplitude up to $A \approx 0.8 x_{0}$, where $\omega \approx 0.4 \omega_{0}$, and then rises for larger amplitudes, reaching $\omega_{0}$ again when $A \approx 2 x_{0}$. Note that the curves depend on simple nondimensional variables and so are quite universal within the approximation of this treatment. It is the delightful simplicity of this result that makes the present discussion worthwhile.

\section{B. Finite shell correctlon}

When the shell is not ideally thin, the flexural rigidity term $\mathrm{c} / \mathrm{m}$ cannot be ignored in the expression for $g$ in (13). It would be possible to carry this term through as a parameter, but for physical interpretation of the results it is necessary to relate it explicitly to the geometry of the shell. Fortunately, this requires consideration only of the small-amplitude linear limit (5) and for this case the solution is well known.

Reissner ${ }^{6}$ has discussed the vibrations of a shallow spherical shell with its edges clamped and has used a variational method to derive an approximate closed expression for the frequency of the lowest mode. In terms of our notation, his result is

$$
\begin{aligned}
\omega_{0}= & 2.98 \frac{h}{r^{2}}\left[\frac{E}{\rho\left(1-v^{2}\right)}\right]^{1 / 2}[1+(1+v) \\
& \left.\times(0.90-0.20(1+v)\}\left(x_{0}^{2} / h^{2}\right)\right]^{1 / 2},
\end{aligned}
$$

where $r$ is the radius of the segment comprising the shell, $x_{0}$ is its central height as before, and $h$ is its thickness. The quantities $E, \rho$, and $v$ refer respectively to the Young's modulus, density, and Poisson's ratio of the shell material.

Rewriting (20) in a form similar to (5), we have

$$
\begin{aligned}
\omega_{0}^{2}= & 8.9\left[\frac{E}{\rho\left(1-v^{2}\right)}\right] \frac{1}{r^{4}}[(1+v) \\
& \left.\times(0.90-0.20(1+v)] x_{0}^{2}+h^{2}\right],
\end{aligned}
$$

where the first term in brackets is derived from membrane stresses and the second is derived from the bending stiffness. Clearly, the second term can be neglected if, and only if, $h<x_{0}$. Comparing (21) with (5), we drive the result

$$
c / m=\alpha \omega_{0}^{2},
$$

where

$$
\alpha=\left[1+(1+v)\{0.90-0.20(1+v)\}\left(x_{0}^{2} / h^{2}\right)\right]^{-1} .
$$

All the material properties except for the Poisson ratio $v$ have now been collected into the mode frequency $\omega_{0}$ and, since $v$ is rarely very different from 0.35 , we have the nearly universal result

$$
\alpha \approx\left[1+0.85\left(x_{0}^{2} / h^{2}\right)\right]^{-1},
$$

which depends solely upon geometrical variables.

Substituting (22) in (13) and using (10), we find that the equation determining $B$ is now the cubic

$$
B^{3}+3 x_{0} B^{2}+\left(\frac{2 x_{0}^{2}}{1-\alpha}+\frac{3}{2} A^{2}\right) B+\frac{3}{2} x_{0} A^{2}=0,
$$

and this is more easily solved numerically than analytically. The physically significant root is that for $\boldsymbol{B}$ closest to zero. The frequency shift $\dot{\phi}$ is then found from the equation

$$
\dot{\phi} \approx\left[3 \omega_{0}(1-\alpha) / 4 x_{0}^{2}\right]\left(\frac{1}{4} A^{2}+B^{2}+2 x_{0} B\right)
$$

and the amplitude change is, as before,

$$
\dot{A} \approx-k A \text {. }
$$

These equations lead to exactly the same results as before in the limit $h \rightarrow 0$. For finite values of the shell thickness, the results have the form shown in Fig. 4, where a value of 0.35

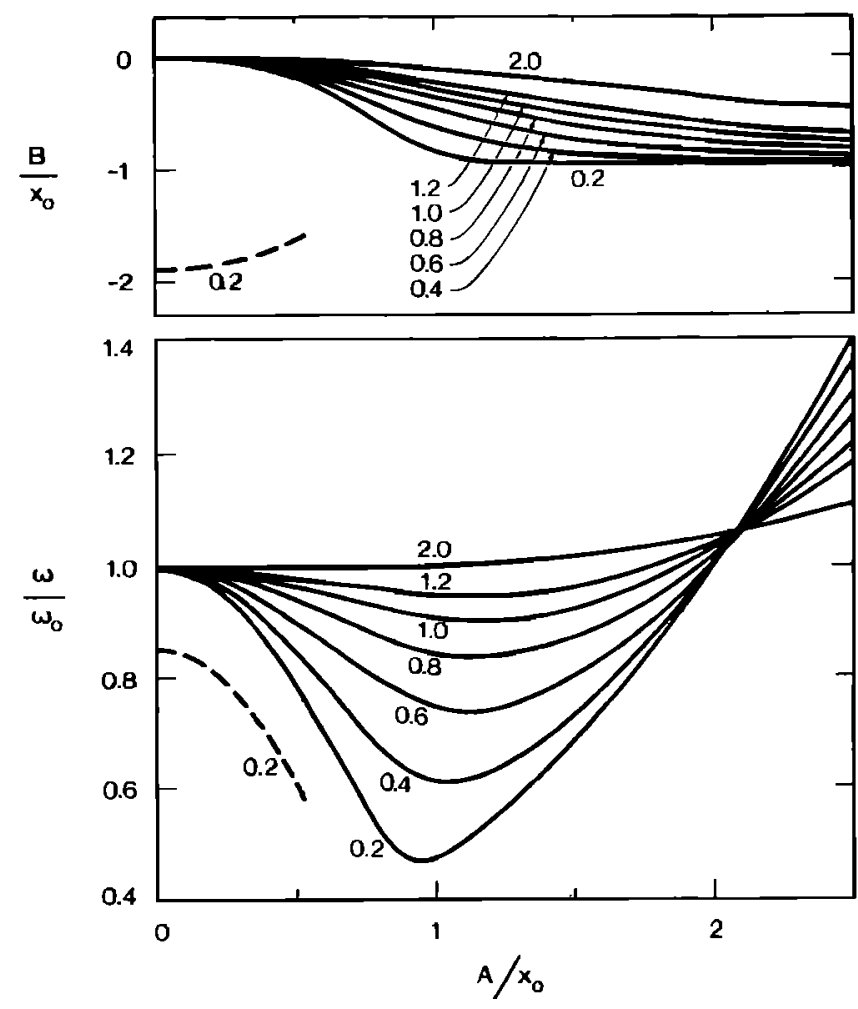

FIG. 4. Calculated motion center $B$ and frequency $\omega$ for vibrations of amplitude $A$ on a spherical-cap shell of dome height $x_{0}$ when the normalized shell thickness $h / x_{0}$ has the value shown as a parameter. The normal mode frequency for small-amplitude vibrations is $\omega_{0}$. The broken curve shows the limited-range behavior of a moderately thin everted shell. 
has been assumed for $v$. With this assumption, the curves are once more universal when expressed in terms of the dimensionless variables $\omega / \omega_{0}$ and $A / x_{0}$, with $h / x_{0}$ as parameter.

We note that for all plate thicknesses $h$ less than about $2 x_{0}$, the frequency falls with increasing amplitude and then rises again to exceed the original value $\omega_{0}$ for $A$ greater than about about $2 x_{0}$. The extent of this frequency shift decreases for increasing shell thickness and, for $h \gtrsim 2 x_{0}$, there is a simple slow increase of $\omega$ with $A$.

For very thin shells $\left(h \leq 0.3 x_{0}\right)$, there is a second equilibrium position with $B<-x_{0}$ but this is stable only for small amplitude vibrations, and for these its mode frequency is below $\omega_{0}$. Shells capable of such eversion are not musically useful since their final state and, hence, final vibration frequency is not reliably controllable.

In the limit when $x_{0} \rightarrow 0$ and the dome becomes flat, we find from (23), (25), and (26) that

$\dot{\phi} \approx \frac{3(1+v)}{16}[0.90-0.20(1+v)] \omega_{0}\left(\frac{A}{h}\right)^{2} \approx 0.16 \omega_{0}\left(\frac{A}{h}\right)^{2}$,

so that the frequency rises as the square of the amplitude. This result also has a universal form, though scaled in terms of $h$ rather than $x_{0}$. It is hardly necessary to plot this simple expression. The apparent anomaly that $\dot{\phi} \rightarrow \infty$ as $h \rightarrow 0$ is accounted for by the fact that, in the absence of any membrane tension, the resonance frequency falls to zero as the stiffiness vanishes. It would be relatively simple to include the effect of residual tension by adding a further linear term to (4) and consequently to $(21)$.

\section{Harmonics and higher modes}

In our discussion, we have used a simple harmonic approximation (7) for the time variation of the motion for the fundamental mode although the force function on the righthand side of $(6)$ is clearly nonlinear. It is a conceptually simple matter to refine this approximation by including harmonics of the fundamental mode. These harmonics have the same spatial pattern as does the fundamental itself and have no connection with the higher normal modes of the vibration, which we shall discuss later. Because of this geometrical similarity, these harmonics are described by the same generalized coordinate $x$ as is the fundamental but we must generalize the time variation (7) and (8) by writing

$$
\begin{aligned}
x= & B+A_{1} \cos \left(\omega_{0} t+\phi_{1}\right)+A_{2} \cos \left(2 \omega_{0} t+\phi_{2}\right) \\
& +A_{3} \cos \left(3 \omega_{0} t+\phi_{3}\right) .
\end{aligned}
$$

Inclusion of both the second and third harmonics is dictated by the physics of the problem.

Application of the procedure leading to Eqs. (10)-(13) then yields not three but rather seven coupled nonlinear equations for the seven quantities $B, \dot{A}_{i}, \dot{\phi}_{i}$. However, a little thought shows that $A_{2}$ and $A_{3}$ are generated nonlinearly from $A_{1}$ so that it is simplest to solve the governing equations by successive approximation using the physically imposed constraints

$$
\phi_{2}=2 \phi_{1}, \quad \phi_{3}=3 \phi_{1} .
$$

The first approximation, neglecting $A_{2}$ and $A_{3}$, yields simply the analysis and results already set out in this paper. The second approximation gives $A_{2}$ and $A_{3}$ as functions of $A_{1}$ and hence of time. It is quite easy to see that, for small amplitudes $A_{1}<x_{0}, A_{2}$ varies as $A_{1}^{2}$ and $A_{3}$ as $A_{1}^{3}$. For $A_{1} \gtrsim x_{0}$ however, the motion becomes more nearly symmetric and $A_{2}$ decreases with increasing $A_{1}$. Indeed, in the thin-shell approximation, $A_{2}$ is zero for $A_{1} \gtrsim 0.8 x_{0}$. In the third approximation, the smaller influence of $A_{2}$ and $A_{3}$ on $A_{1}$ is taken into account. Clearly, the harmonic structure of each modal vibration changes in a rather complex way with time after a vigorous blow to the gong.

Higher normal modes of the gong are another matter altogether. They differ from one another in geometry-indeed they are orthogonal-and their frequencies are in no simple relationship. The interaction between membrane forces and stiffness forces for these modes should be similar to that for the fundamental mode, but a correspondingly. simple analysis does not seem possible because the plane configuration does not occur as an intermediate state in the motion. Because of their orthogonality, the modes do not couple appreciably to one another and it is reasonable to treat them independently.

Because of the limited importance of the Chinese gong problem, it is hardly worthwhile to pursue these elaborations of the theory in detail here.

\section{Validity}

Our concern here has been to develop a set of rather generally applicable approximate formulas rather than to investigate a well-defined situation with precision. It is important therefore to consider briefly the major assumptions and approximations to see how these might limit the accuracy and generality of the results.

The shell we discussed was assumed to be sufficiently shallow that it was able to pass through an intermediate plane configuration without buckling. This is an important consideration and at the same time difficult to quantify without elaborate investigation. We shall not consider this further except to note its importance in defining a limit to the shell aspect ratio $r / x_{0}$ to which the theory is applicable.

We also assumed the shell to be a spherical cap though the word "quasispherical" is used in the title. The behavior of all shells of nearly spherical shape should be similar, since the range of shape variation possible in a very shallow shell is not large. The shell shape will, however, influence the mode shape and with it the exact scaling of the amplitude parameter $A / x_{0}$. For small shape variations, the rescaling should not be significant.

It is relevant to inquire the limit of validity of the results in range of the amplitude parameter $A / x_{0}$. The next nonlinear term in the membrane force (1) involves $\left(x+x_{0}\right)^{5}$ and it is the magnitude of this term which must be considered in comparison with the cubic term. The form (1) for the restoring force is exact, in an energy average sense, provided the shape of the cap remains spherical as it vibrates. In fact, the radial parts of the eigenfunctions are combinations of Bessel functions and modified Bessel functions of order zero, as is taken into account explicitly in derivation of the mode frequency 
$\omega_{0}$ in (20). While it is not expected that the fifth power terms would influence the results significantly over the amplitude range depicted in Fig. 4, it is difficult to make an estimate other than by an explicit solution retaining terms to this order. Such a solution does not appear to be available in the literature, but it is relevant to remark that our results agree almost exactly with those plotted over a more limited range by Grossman et al. ${ }^{2}$ for the two cases $x_{0}=0,0<A / h<2$ and $h / x_{0}=0.5,0<A / x_{0}<1$ (in our notation), which are the only explicit results given. The general behavior illustrated in Fig. 4 is also similar to that calculated by Leissa and $\mathrm{Kadi}^{3}$ for rectangular shells.

\section{CONCLUSIONS}

For the small gong discussed in our earlier paper, ${ }^{1}$ the striking shell is about $10 \mathrm{~cm}$ in diameter, the material thickness of the shell is about $0.7 \mathrm{~mm}$, and the cap height $x_{0}$ is about $1 \mathrm{~mm}$, though the curvature departs from spherical in such a way that the equivalent spherical $x_{0}$ may be somewhat less than this. Assuming a value of $h / x_{0}$ near 0.7, the curves of Fig. 4 predict a maximum frequency depression of about $20 \%$ for an amplitude $A$ somewhat greater than $x_{0}$. Since one semitone corresponds to a frequency change of approximately $6 \%$, this implies a maximum pitch glide of around three semitones, which is rather more than the aurally judged glide but in acceptable agreement with experiment in view of the uncertainty of the observation and the deviation from spherical curvature.

It is interesting to note that the shape of the curves in Fig. 4 implies that the total pitch glide is fairly stable for amplitudes in the range $0.8 x_{0}$ to $1.5 x_{0}$, with a smaller glide for less energetic excitation. This too agrees with experience. It does not appear possible, with the rather light soft stick provided with these gongs, to achieve an amplitude suffi- ciently large to exhibit the reversing glide predicted by the theory.

In the case of the larger gong, the diameter of the central section is about $15 \mathrm{~cm}$ and the material thickness is about 1 $\mathrm{mm}$. A vigorous blow can give a vibration amplitude approaching $1 \mathrm{~mm}$ so that, by (28), a downward pitch glide of several semitones is predicted, in agreement with observation.

In summary, this discussion reveals clearly the design parameters upon which the pitch glide phenomenon depends. Apart from the excitation amplitude, these are purely geometric and reduce essentially to the ratio of the shell thickness $h$ to the shell center offset $x_{0}$. The fundamental frequency of the gong also depends upon $h, x_{0}$ and the gong central radius $r$, together with the elastic constants of the gong metal, as set out in Eq. (20). It is the remarkable simplicity of these results that makes the approximate treatment set out in this paper worthwhile.

'T. D. Rossing and N. H. Fletcher, "Nonlinear vibrations in plates and gongs," J. Acoust. Soc. Am. 73, 345-351 (1983).

${ }^{2}$ P. L. Grossman, B. Koplik, and Y-Y. Yu, "Nonlinear vibrations of shallow spherical shells," J. Appl. Mech. 36, 451-458 (1969).

${ }^{3}$ A. W. Leissa and A. S. Kadi, "Curvature effects on shallow shell vibrations," J. Sound Vib. 16, 173-187 (1971).

"N. H. Fletcher, "Axisymmetric wave propagation on a conical shell," $\mathrm{J}$. Acoust. Soc. Am. 72, 250-254 (1982).

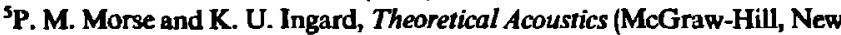
York, 1968), pp. 838-840.

${ }^{6}$ E. Reissner, "On vibrations of shallow spherical shells," J. Appl. Phys. 17, 1038-1042 (1946). 